\title{
The Impact of Obesity on In Vivo Human Skeletal Muscle Function
}

\author{
Nicola A. Maffiuletti • Sébastien Ratel • Alessandro Sartorio • Vincent Martin
}

Published online: 12 June 2013

(C) Springer Science+Business Media New York 2013

\begin{abstract}
Despite the considerable efforts that have been made to characterize in vivo human skeletal muscle function in the last 20 years, there is still controversy about whether obesity affects muscle performance in people of different ages. We therefore reviewed the available literature to determine the impact of obesity on skeletal muscle strength and fatigue. Obese individuals have (i) higher absolute muscle strength, (ii) lower strength per unit body mass, (iii) a similar strength to total fat-free mass ratio and (iv) a similar/higher strength to muscle size ratio compared to their nonobese peers. These results suggest that obesity does not negatively affect the intrinsic muscle contractile properties. Moreover, the available evidence does not show differences in muscle fatigue between obese and nonobese individuals. Therefore, factors such as the handicapping effect of excess fat mass and/or impaired motor coordination may account for the poor physical performance of obese people of all ages.
\end{abstract}

\footnotetext{
N. A. Maffiuletti $(\bowtie)$

Neuromuscular Research Laboratory, Schulthess Clinic, Lengghalde 2 ,

8008 Zurich, Switzerland

e-mail: nicola.maffiuletti@kws.ch

S. Ratel · V. Martin

Laboratory of Metabolic Adaptations to exercise in Physiological and Pathological conditions (AME2P, EA 3533), Blaise Pascal

University, BP 10448, 63000 Clermont-Ferrand, France

S. Ratel

e-mail: Sebastien.RATEL@univ-bpclermont.fr

V. Martin

e-mail: Vincent.MARTIN@univ-bpclermont.fr

\author{
A. Sartorio \\ Experimental Laboratory for Auxo-endocrinological Research \& \\ Division of Metabolic Diseases and Auxology, Istituto Auxologico \\ Italiano, Via Ariosto 13, \\ 20145 Milan \& Piancavallo, Italy \\ e-mail: sartorio@auxologico.it
}

Keywords Muscle strength $\cdot$ Muscle power $\cdot$ Muscle fatigue · Dynamometer $\cdot$ Obesity $\cdot$ Muscle function

\section{Introduction}

Although the cardiovascular and metabolic consequences of obesity have been studied extensively over the last two decades, less attention has been paid to investigating the impact of obesity on in vivo human skeletal muscle function. This is surprising in light of the fact that obese people have considerable functional limitations [1] and an increased prevalence of health problems, which are due, at least in part, to insufficient levels of skeletal muscle strength and power in relation with their excessive body mass (i.e., overall inadequate skeletal muscle function).

The two main attributes of in vivo human skeletal muscle function are maximal voluntary strength and power, which can be objectively and validly evaluated during all-out isometric (strength only), concentric or eccentric contractions, and whose outcomes can be expressed in absolute units (absolute strength/power) and/or as a function of body mass or muscle size (relative strength/power). The physiological determinants of muscle strength and power are classically categorized as neural and muscular. Neural factors mainly include activation patterns of both agonist and antagonist muscles, while the two major muscular determinants are muscle size and muscle fiber-type distribution. Evaluating muscle strength/power and their physiological determinants in obese subjects is challenging, because of large body size, that complicate subject positioning in conventional dynamometers or MRI scanners, and because of the presence of large amounts of subcutaneous fat, that partially invalidate neuromuscular assessment techniques such as surface electromyography, ultrasonography and magnetic/electrical stimulation of peripheral nerves. Despite the considerable efforts that have been made to characterize in vivo human skeletal muscle 
function in the last 20 years (see e.g.,[2••]), there is still controversy about whether obese people of different ages are able to generate the same strength or power than their lean peers.

To our knowledge, the effect of obesity on muscle fatigue, which - besides muscle strength and power - represents an important link to normal daily-living tasks, is also poorly documented in the literature. This information is nonetheless essential since several daily (e.g., stair climbing, walking), as well as physical activities involve repetitive contractions of the lower limb muscles. Greater fatigue in these muscles could thus be seen as a limiting factor for motor performance. Owing to the higher proportion of fast-fatigable fibers described in obese human skeletal muscles [3, 4], it could be envisaged that obese people would experience an enhanced fatigability as compared to their lean counterparts. Intuitively, one can also expect that the high levels of power required by obese subjects to move their massive body during ambulatory and sport activities may lead to enhanced fatigability.

The main objectives of this review paper are (i) to reexamine the impact of obesity on in vivo human skeletal muscle strength/power and fatigue by reviewing previous studies conducted on children, young adults, and elderly subjects, (ii) to discuss the main implications of these findings in relation with functional disabilities and the eventual occurrence of diseases/pathologies, and (iii) to provide possible perspectives for future research in this area.

In the first part of the review, we only included studies in which human skeletal muscle strength/power (hereafter referred to as strength only, both for the sake of clarity and because power has been rarely investigated) and fatigue have been objectively evaluated (i.e., by means of dynamometry), so as to exclude assessments with poor methodological validity and to minimize the influence of coordination on musclerelated outcomes. The literature search was conducted using PubMed database throughout the years 1970-2013 and using the following keywords: "obesity", "body mass index", "strength", "power", "fatigue", "muscle" and "function". We excluded studies whose experimental design lacked a clear definition of obesity, to avoid confusion between overweight and obesity, and with an imprecise description of muscle strength and fatigue assessment protocols.

\section{Impact of Obesity on Muscle Strength}

Table 1 summarizes the studies that have examined the impact of obesity on muscle strength and/or power by comparing nonobese and obese individuals of similar chronological age. Overall, the literature indicates that the comparison of muscle strength between obese and nonobese people is affected by the specificity of the normalization procedure, muscle groups investigated, muscle length at which strength measurements were conducted, age and the amount of habitual physical activity.

Taken as a whole, data indicate that obese people have higher absolute muscle strength, but lower relative values than nonobese subjects when strength is expressed per unit body mass [5••, 6-13]. On the contrary, obese individuals have a similar strength to total fat-free mass ratio $[6,8,10$, 12-14] and a similar or higher strength to muscle size ratio compared to their lean counterparts $[5 \cdot \bullet, 15,16]$. These results suggest that obesity does not seem to negatively affect the intrinsic muscle contractile properties ("muscle quality"). Consequently, other factors, such as the handicapping effect of excess fat mass, and/or impaired motor coordination [17] may account for the reduced motor performance of obese people, especially for complex motor tasks that require body mass support or mobilization.

The higher absolute muscle strength in obese people seems to be more frequently reported in prepubescent children, adolescents and young adults, than in elderly people. For instance, we [5••, 6, 8, 14] and others [13] have reported significantly higher absolute cycling peak power and maximal isometric/isokinetic strength of knee extensor muscles in obese children/adolescents as compared to controls. Furthermore, Miyatake et al. [11] showed that absolute isometric strength of the knee extensors was higher in 20 to $60 \mathrm{yr}$ old obese adults compared to nonobese controls, while no obesity-related difference were detected in subjects over $60 \mathrm{yr}$. In elderly subjects, however, data are limited and highly controversial. Although some authors reported a reduced absolute muscle strength in elderly obese subjects [18], others reported similar and even higher muscle strength in obese compared to lean elderly subjects [11, 19]. Part of these controversies could be attributed to differences in the amount of habitual physical activity achieved during aging. For instance, Rolland et al. [19] showed that active obese elderly people produced higher relative strength per unit muscle size than nonobese, while sedentary obese elderly had similar muscle strength compared to nonobese. Consequently, favorable adaptations to excess body mass on muscle function might depend on the sustenance of sufficient levels of physical activity during aging.

Comparison of muscle strength between obese and nonobese populations could also differ according to the muscle group investigated. Specifically, the higher absolute muscle strength of obese individuals could be more relevant for larger muscle groups involved in lifting and/or moving the body (i.e., knee and trunk extensors). Whilst some studies found higher absolute isokinetic/isometric strength of knee/trunk extensors in obese subjects [7,9], others have reported no significant difference in maximal isokinetic torque of knee/elbow flexors between obese and nonobese people $[7,9,15]$. Thus, the extra-load associated with severe 


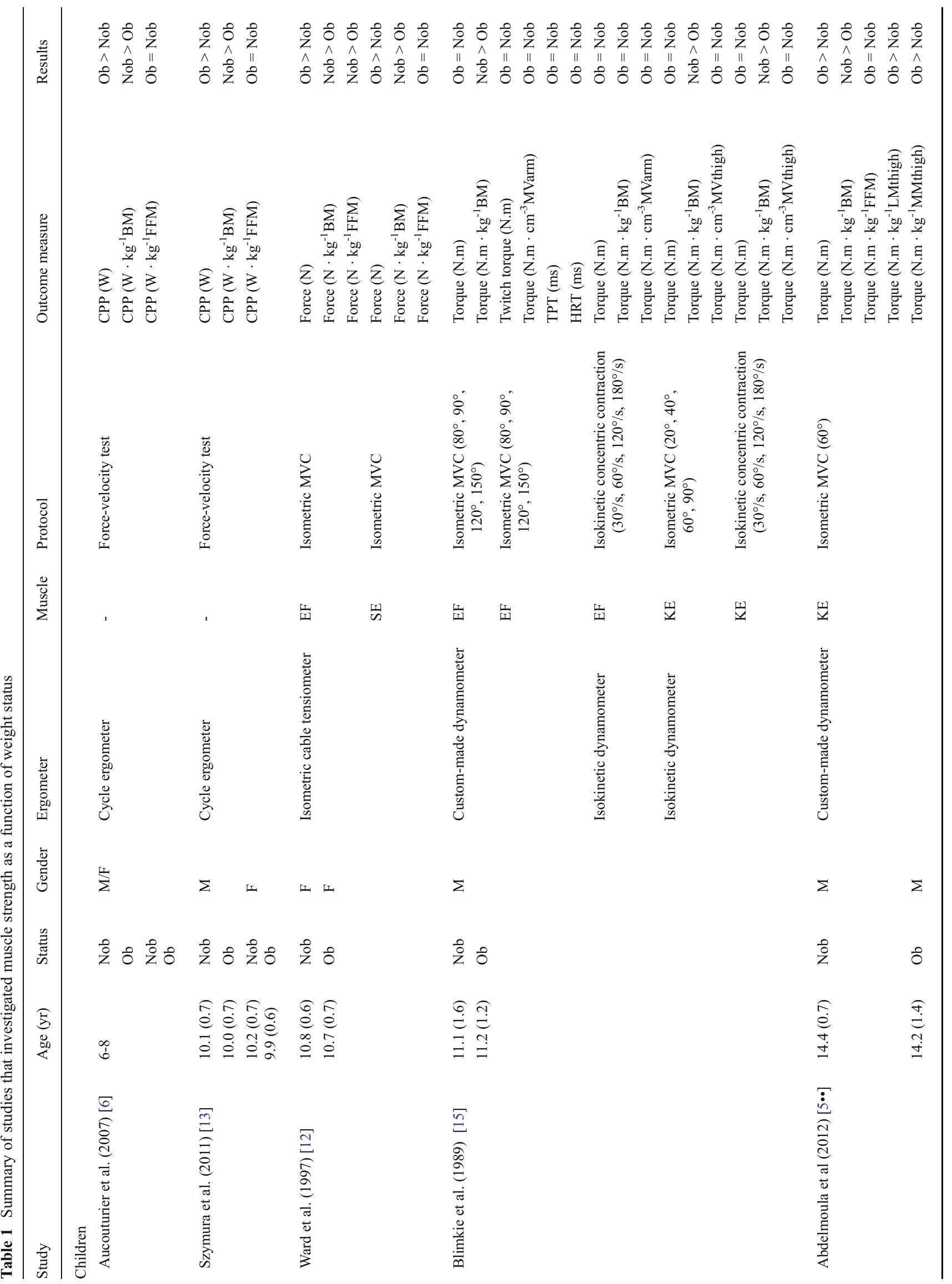




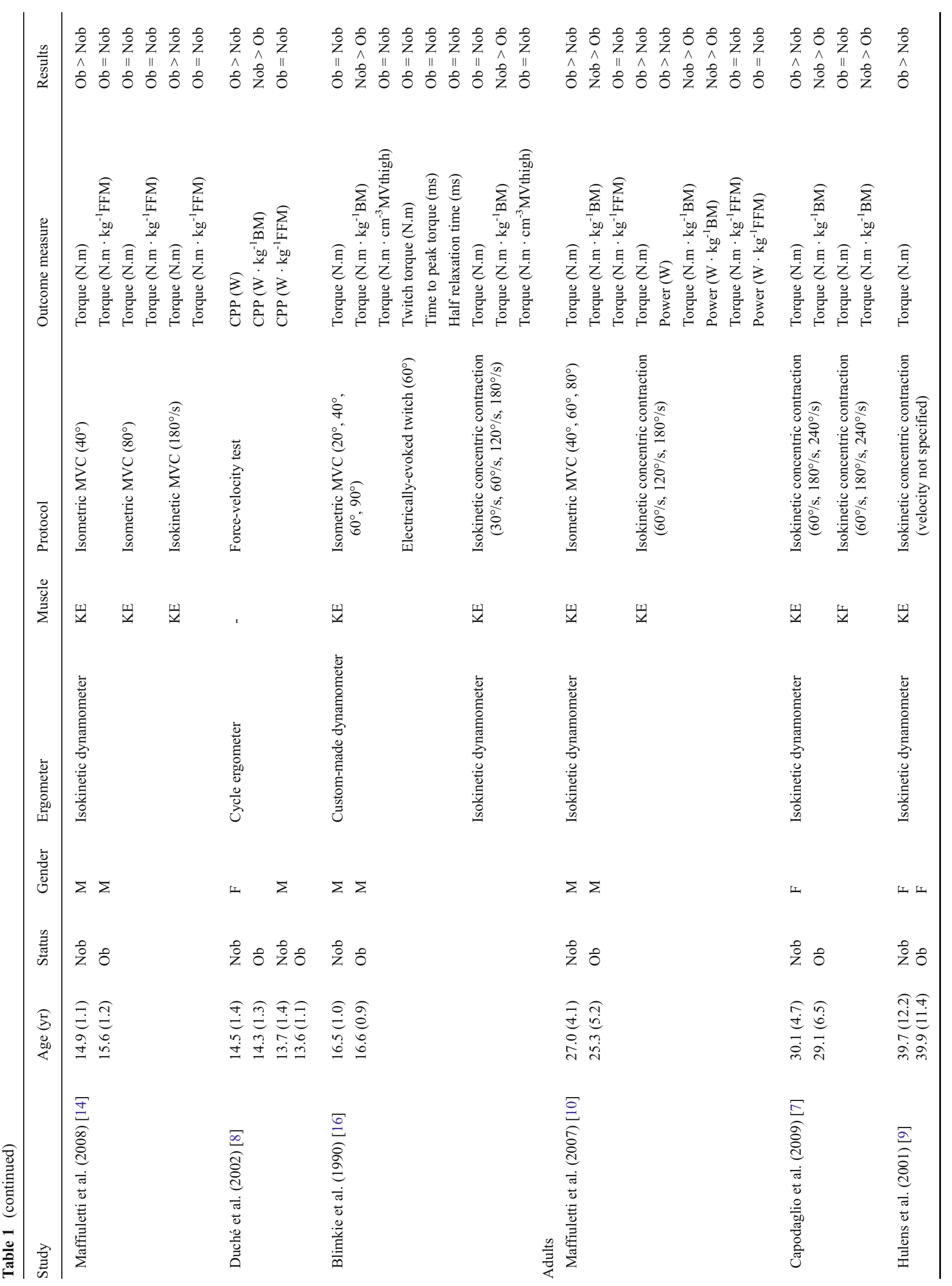




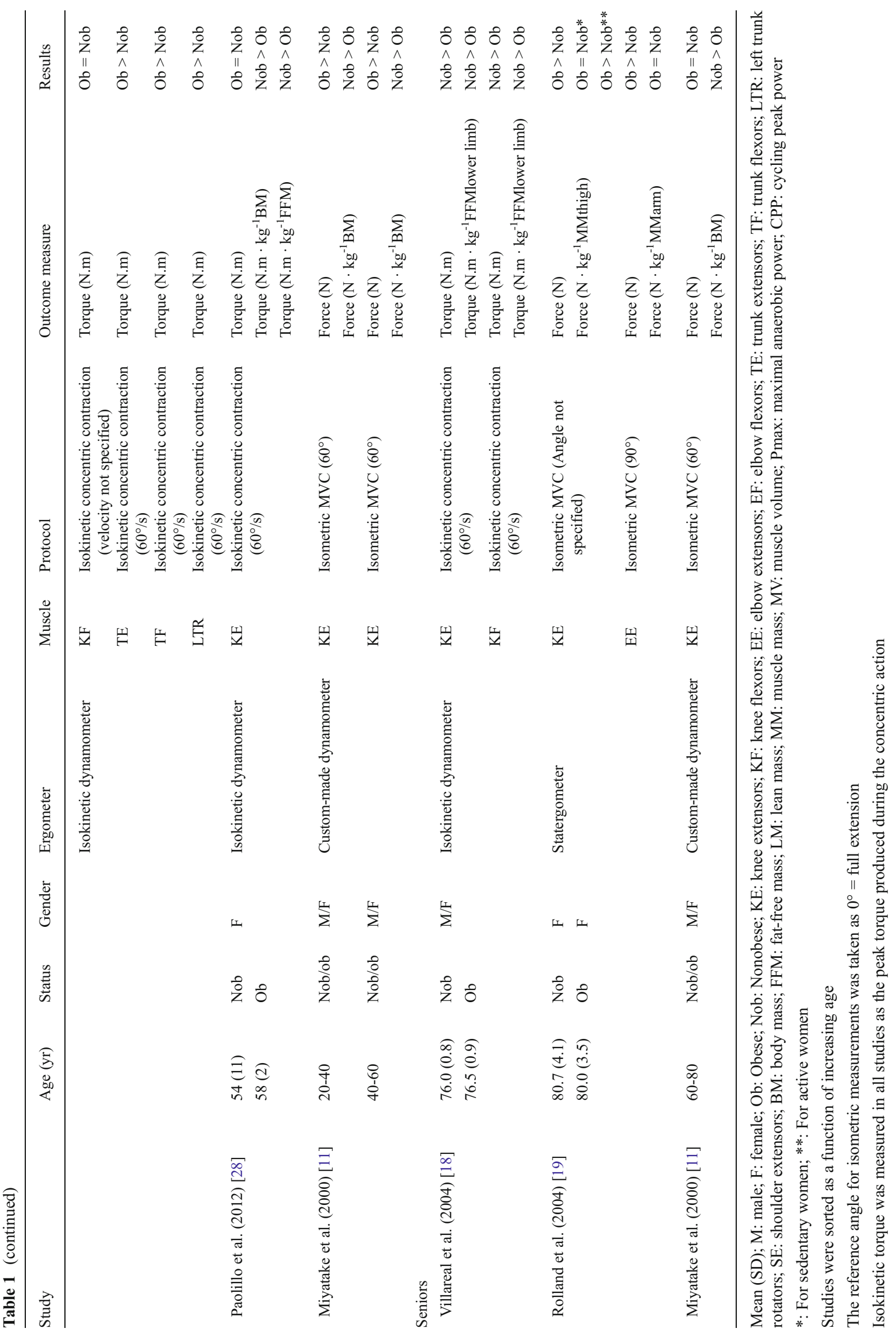


obesity could act as a chronic training stimulus generating favorable muscle adaptations. This contention has support from research showing greater absolute amounts of fat-free mass in obese people compared to lean controls $[5 \bullet \bullet, 6,8,10$, 14]. However, caution should be taken when considering this hypothesis as no significant difference was observed in thigh muscle mass (determined by dual-energy X-ray absorptiometry) and muscle cross-sectional area of knee extensors (measured by computed axial tomography) between obese and nonobese adolescents $[5 \cdot \bullet, 16]$. This led us to acknowledge that the hypothetic cause and effect relationship between excess body mass and increased skeletal muscle mass ("muscle quantity") is far from being established.

Alternatively, one may conjecture that the duration of exposition to overloading, i.e., duration of obesity, could also influence skeletal muscle mass (and thus physical function). However, no data is currently available to prove this assumption. Conversely, the findings of Blimkie et al. [16] and Abdelmoula et al. [5••] are in accordance with the data from Sitnick et al. [20] showing that mice chronically fed with a high-fat diet demonstrated an impaired ability of the skeletal muscle to hypertrophy in response to increased mechanical loading. Similarly, it has been shown in rats that obesity impaired the regulation of troponin $\mathrm{T}$ expression and hence altered the ability of skeletal muscle to respond appropriately to the increased body mass [21•]. Furthermore, no significant difference was found in the intrinsic contractile properties of knee extensors between obese and nonobese adolescents [16]. Consequently, it appears legitimate to speculate that the higher absolute strength of knee extensors in obese people could be mainly accounted for by neural factors, which might include higher agonist activation, lower antagonist muscle coactivation and/or an increased contribution of synergistic muscles. However, to date, only Blimkie et al. [16] compared voluntary muscle activation (twitch interpolation technique) of the knee extensors between obese and nonobese adolescents and reported significantly lower activation scores in obese adolescents. The authors suggested that this activation deficit may account for the lower muscle strength to body mass ratio and the reduced motor performances in obese adolescents. To the best of our knowledge, no data are available regarding the impact of obesity on antagonist muscle coactivation and synergistic muscle recruitment.

Other factors may be put forward to explain the increased absolute strength of obese people. Adaptations in skeletal muscle architecture could potentially contribute to an increased force-generating capacity without significant changes in muscle volume/size. However, no data is currently available to verify this assumption, probably because of the relative inaccessibility of muscle fascicles with ultrasonography, especially for the deepest muscles, in subjects who are obese. Adaptations in musculo-tendinous stiffness could also have a favorable effect on the rate of force development and thus on the muscle power produced during "explosive" movements, and during eccentric contractions. Interestingly, it has recently been reported that weight-related additional loading resulted in a greater stiffness of the triceps surae musculo-tendinous unit in obese children [22•] and postmenopausal women [23]. This higher musculo-tendinous stiffness in obese people, which could be the consequence of fat infiltration into skeletal muscle and increased inter-muscular adipose tissue [23], could partly explain their higher absolute and relative (i.e., per unit muscle size) muscle power. Finally, differences in muscle performance between obese and nonobese individuals could also be mediated by muscle length specificities, and thus by the joint angle at which strength measurements were made. Specifically, Maffiuletti et al. [14] showed that absolute knee extension isometric torque was significantly higher in severely obese adolescents compared to lean controls at short $\left(40^{\circ}\right.$ of knee flexion) but not at long $\left(80^{\circ}\right)$ muscle length. Accordingly, severely obese subjects would present an advantage at short rather than at long muscle length because they would probably deliberately limit their range of motion during daily activities involving deep knee flexion, due to the excessive stress acting on the articular joint surfaces. This would in turn result in favorable but specific neuromuscular adaptations at short muscle length. Additionally, one may suggest that this angle specificity could also reflect the need to produce high muscle strength at short muscle lengths (i.e., when lower limbs are extended) to maintain a standing posture, whose regulation is harder in obese people due to body mass excess (see [24••] for review). However, this is speculation and further research is needed before definitive conclusions can be drawn on this issue.

\section{Impact of Obesity on Muscle Fatigue}

To date, only four studies have addressed objectively the issue of muscle fatigue and its etiology in obese subjects, but these studies yielded conflicting results (Table 2). Maffiuletti et al. [10] evaluated the voluntary torque loss during 50 concentric knee extensions in adult obese and lean men, and observed a greater fatigue magnitude in the former. In order to gain insight into the origin of this enhanced fatigability, Maffiuletti et al. [10] additionally evaluated the profile of torque decrement during a series of intermittent knee extensions evoked by electrical stimulation. No difference was observed between the two groups of adult men, indirectly suggesting that central factors may have accounted for the enhanced fatigability of obese subjects, and peripheral factors did not differ between populations, thereby rejecting the hypothesis of a different muscle fiber-type distribution between obese and lean adults. Nevertheless, the potential implication of central factors in the development of fatigue still needs to be measured directly in obese subjects. Minetto et al. [2••] recently investigated the 


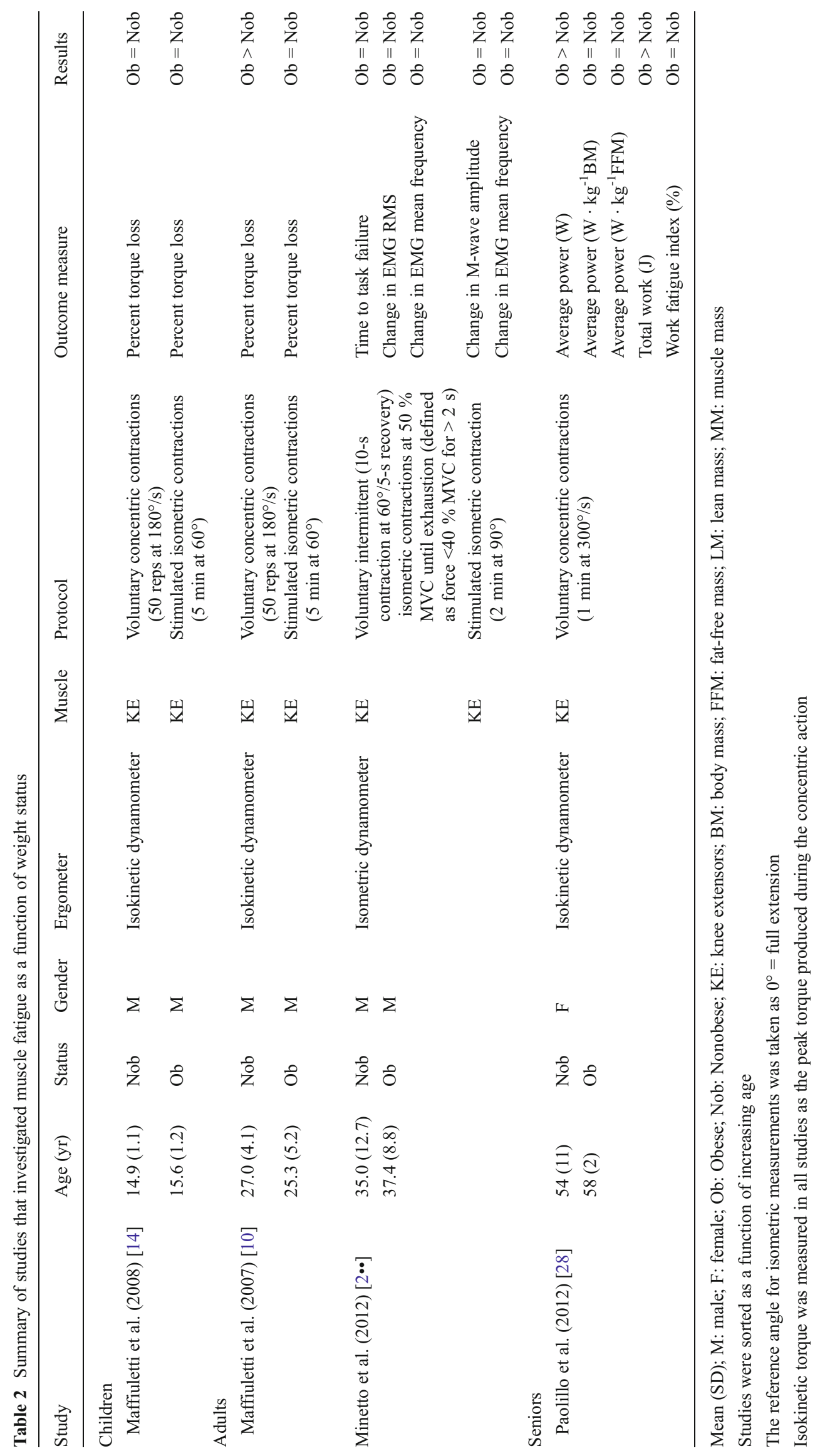


physiological manifestations of fatigue over the course of intermittent voluntary and sustained stimulated contractions of the knee extensors, but failed to demonstrate any difference in fatigue-induced electromyographic and mechanical alterations between obese and lean adults. Interestingly, this previous study demonstrated that surface electromyographic signal detection and electrical stimulation of the quadriceps muscle are feasible even in severely obese subjects.

Maffiuletti et al. [14] repeated their study in obese and lean male adolescents but reported conflicting results since no difference in fatigability was observed between the two groups, both with voluntary and stimulated fatigue protocols. An effect of age and/or duration of obesity on muscle fatigability cannot be ruled out. In fact, Sartorio et al. [25] revealed that subjective fatigue perception, as measured with the Fatigue Severity Scale [26] in obese adults, was influenced both by age and obesity level, resulting in lower scores (i.e., lower fatigue perception) in younger $(<45 \mathrm{yr})$ than in older subjects $(>45 \mathrm{yr})$ and in patients with a BMI lower than $40 \mathrm{~kg} / \mathrm{m}^{2}$ compared to those with a BMI higher than $40 \mathrm{~kg} / \mathrm{m}^{2}$. However, counter-arguments can be also put forward. Indeed, Levinger et al. [27] compared the psychological responses of obese and nonobese subjects to a resistance training session and reported no significant difference of subjective fatigue scores between obese and lean women but a higher fatigue score (i.e., higher fatigability) in lean male subjects as compared to their obese counterparts, which is contradictory to the results of Sartorio et al. [25]. Nevertheless, the results reported for women are consistent with those of Paolillo et al. [28] who recently compared the fatigability of normal-weight and obese women and did not observe any significant difference in work decrement over the course of a 1-min set of voluntary concentric knee extensions.

\section{Implications}

Obese people have higher absolute muscle strength than nonobese, regardless of age. When absolute strength is expressed as a function of body mass, however, obese subjects present lower strength than their lean counterparts. These results have multiple implications for people who are obese, in particular with respect to physical functioning during daily-living activities and overall quality of life.

Insufficient muscle strength of obese subjects in relation with their markedly increased body mass is responsible, at least in part, for functional limitations in performing the common activities of daily life, including work capacity [29]. For example, low relative strength, combined with poor static and dynamic postural stability and reduced sensory integration in obese individuals [30], can increase the risk of falling and stumbling during ambulation [31,32], and even more so for the elderly obese. The obesity-related quadriceps weakness reported at long muscle length [14] could also represent a serious limitation in everyday life and eventually a risk factor for musculoskeletal injuries. Daily activities involving lengthening contractions of the quadriceps with wide range of motion (such as kneeling and crouching) can represent an excessive challenge for people who are obese, thereby limiting their physical functioning.

Obese people exert high absolute forces to support and move their massive body during common activities of daily living, such as walking [33]. This may lead to abnormal joint loading [34], gait mechanics [35] and joint alignment (especially at the knee joint) [36], which could represent a possible pathway for the pathogenesis and progression of knee osteoarthritis [37] and of orthopedic complications in general, regardless of age [38]. Obesity is a major risk factor for knee osteoarthritis $[33,37]$, with a relative ratio of 4.4 in women and 2.8 in men [37]. The possible relation between obesity, insufficient relative muscle strength, and osteoarthritis is, however, far from being established.

\section{Perspectives}

This review article raises several important questions. With respect to the in vivo evaluation of skeletal muscle performance, the impact of obesity on the following crucial attributes of human muscle function remains to be determined:

- eccentric muscle strength (especially at long muscle length), which has been completely overlooked in previous research;

- dynamic muscle power, particularly for multi-joint closed-chain exercises (e.g., leg press, half squat), which is better related to global physical function than pure muscle strength;

- stretch-shortening cycle performance, to evaluate the energy storage capacity of the series elastic components and the contribution of the stretch reflex;

- validity and reliability of skeletal muscle function outcomes, which could be worst in obese compared to nonobese individuals due to poor coordination, body stabilization, and motivation;

- muscular and neural adaptations induced by long-term excessive body mass, such as chronic changes in muscle mass, muscle fiber-type distribution, spinal reflex and cortico-spinal excitability.

- owing to the specificity of psychological responses of obese people to voluntary physical exercise [39], both peripheral and central factors contributing to the development of muscle fatigue should be systematically evaluated in obese subjects;

- neuromuscular, physical and mental fatigue induced by physical exercise as well as by common tasks of daily living. 
More work is also required to demonstrate the potential cause and effect relationship between poor skeletal muscle function and physical dysfunction in obese subjects of different ages and sexes. This will, in turn, help clarifying whether or not obese people require strength training, in addition to low-intensity aerobic and/or high-intensity intermittent exercise, as a part of their multicomponent intervention for managing obesity. Since the prevalence and incidence of obesity are progressively increasing and do not seem to slow down in the next decades, physical rehabilitation of obese subjects will represent a relevant challenge for all the clinicians treating this social disease. Taking into account the progressive age-related negative influences of weight excess, it is mandatory to adequately deal with the treatment and rehabilitation of childhood obesity, when the weight excess has not negatively affected skeletal muscle function and the ability to perform the common daily activities. Adapted physical activities aimed to preserve (or increase) fat-free mass, tailored for the single obese subject and taking into account his/her physical disabilities and associated comorbidities, and combined with adequate nutritional intakes (also adapted to the different life periods), need to be defined in a better way in order to guarantee the more longlasting level of autonomy and health for these subjects.

\section{Conclusion}

In summary, obese people have higher absolute muscle strength than lean peers but lower strength per unit body mass. Conversely, they have a similar strength to total fat-free mass ratio and a similar/higher strength to muscle size ratio compared to nonobese individuals. These results suggest that obesity does not seem to negatively influence the intrinsic force-generating capacity of skeletal muscles. However, there are some specificities of obese muscles due to habitual physical activity levels and/or to long term adaptive changes, e.g., at short lengths and for antigravity muscles (probably mediated by neural adaptations) that require further investigation. The issue of fatigability in obese subjects is highly controversial as too few objective evaluations have been conducted to draw firm conclusions, but the available evidence does not show differences in muscle fatigue between obese and nonobese individuals. The respective influences of age, duration of obesity and gender on muscle fatigability remain to be clarified.

\section{Compliance with Ethics Guidelines}

Conflict of Interest Nicola A. Maffiuletti declares that he has no conflict of interest.

Sébastien Ratel declares that he has no conflict of interest.

Alessandro Sartorio declares that he has no conflict of interest.

Vincent Martin declares that he has no conflict of interest.
Human and Animal Rights and Informed Consent This article does not contain any studies with human or animal subjects performed by any of the authors.

\section{References}

Papers of particular interest, published recently, have been highlighted as:

- Of importance

•. Of major importance

1. Evers Larsson U, Mattsson E. Functional limitations linked to high body mass index, age and current pain in obese women. Int $\mathrm{J}$ Obes Relat Metab Disord. 2001;25:893-9.

2. •- Minetto MA, Botter A, Sprager S, et al. Feasibility study of detecting surface electromyograms in severely obese patients. J Electromyogr Kinesiol. 2013;23(2):285-95. This study proves that the detection of surface electromyographic signals is feasible in severely obese subjects and that fatigue manifestations do not differ between obese and nonobese individuals.

3. Kriketos AD, Baur LA, O'Connor J, et al. Muscle fibre type composition in infant and adult populations and relationships with obesity. Int J Obes Relat Metab Disord. 1997;21:796-801.

4. Wade AJ, Marbut MM, Round JM. Muscle fibre type and aetiology of obesity. Lancet. 1990;335:805-8.

5. •-Abdelmoula A, Martin V, Bouchant A, et al. Knee extension strength in obese and nonobese male adolescents. Appl Physiol Nutr Metab. 2012;37:269-75. In this comparative study we demonstrated that obese adolescent boys have higher absolute and relative isometric knee extension strength than nonobese boys.

6. Aucouturier J, Lazaar N, Dore E, et al. Cycling peak power in obese and lean 6- to 8-year-old girls and boys. Appl Physiol Nutr Metab. 2007;32:367-71.

7. Capodaglio P, Vismara L, Menegoni F, et al. Strength characterization of knee flexor and extensor muscles in Prader-Willi and obese patients. BMC Musculoskelet Disord. 2009;10:47.

8. Duche P, Ducher G, Lazzer S, et al. Peak power in obese and nonobese adolescents: effects of gender and braking force. Med Sci Sports Exerc. 2002;34:2072-8.

9. Hulens M, Vansant G, Lysens R, et al. Study of differences in peripheral muscle strength of lean versus obese women: an allometric approach. Int J Obes Relat Metab Disord. 2001;25:676-81.

10. Maffiuletti NA, Jubeau M, Munzinger U, et al. Differences in quadriceps muscle strength and fatigue between lean and obese subjects. Eur J Appl Physiol. 2007;101:51-9.

11. Miyatake N, Fujii M, Nishikawa $H$, et al. Clinical evaluation of muscle strength in 20-79-years-old obese Japanese. Diabetes Res Clin Pract. 2000;48:15-21.

12. Ward DS, Trost SG, Felton G, et al. Physical activity and physical fitness in African-American girls with and without obesity. Obes Res. 1997;5:572-7.

13. Szymura J, Gradek J, Maciejczyk M, et al. The anaerobic capacity in obese children. Gastroenterol Pol. 2011;18:147-53.

14. Maffiuletti NA, Jubeau M, Agosti F, et al. Quadriceps muscle function characteristics in severely obese and nonobese adolescents. Eur J Appl Physiol. 2008;103:481-4.

15. Blimkie CJ, Ebbesen B, MacDougall D, et al. Voluntary and electrically evoked strength characteristics of obese and nonobese preadolescent boys. Hum Biol. 1989;61:515-32.

16. Blimkie CJ, Sale DG, Bar-Or O. Voluntary strength, evoked twitch contractile properties and motor unit activation of knee extensors in 
obese and non-obese adolescent males. Eur J Appl Physiol Occup Physiol. 1990;61:313-8.

17. D'Hondt E, Deforche B, Vaeyens R, et al. Gross motor coordination in relation to weight status and age in 5- to 12-year-old boys and girls: a cross-sectional study. Int J Pediatr Obes. 2011;6:e556-64.

18. Villareal DT, Banks M, Siener C, et al. Physical frailty and body composition in obese elderly men and women. Obes Res. 2004;12:913-20.

19. Rolland Y, Lauwers-Cances V, Pahor M, et al. Muscle strength in obese elderly women: effect of recreational physical activity in a cross-sectional study. Am J Clin Nutr. 2004;79:552-7.

20. Sitnick M, Bodine SC, Rutledge JC. Chronic high fat feeding attenuates load-induced hypertrophy in mice. J Physiol. 2009;587:575365 .

21. - Schilder RJ, Kimball SR, Marden JH, Jefferson LS. Body weightdependent troponin $\mathrm{T}$ alternative splicing is evolutionarily conserved from insects to mammals and is partially impaired in skeletal muscle of obese rats. J Exp Biol. 2011;214:1523-32. This animal study shows that obesity affects the expression of skeletal muscle troponin $T$ (a component of the thin filament regulating actinmyosin interaction and modulating force output). This is a candidate mechanism for muscle weakness and reduced mobility in obesity.

22. • Lambertz D, Souza TO, Canon F, et al. Influence of overweight on the active and the passive fraction of the plantar flexors series elastic component in prepubertal children. J Appl Physiol. 2013;114:73-80. This study shows that triceps surae musculotendinous stiffness is greater for overweight than lean children due to a concomitant increase in the stiffness of the series elastic component passive fraction and in the articular structures.

23. Faria A, Gabriel R, Abrantes J, et al. Triceps-surae musculotendinous stiffness: relative differences between obese and non-obese postmenopausal women. Clin Biomech (Bristol Avon). 2009;24:866-71.

24. •- Tsiros MD, Coates AM, Howe PR, et al. Obesity: the new childhood disability? Obes Rev. 2011;12:26-36. An excellent review of the effects of obesity on physical functioning and disability in children.

25. Sartorio A, Fontana P, Trecate L, Lafortuna CL. Short-term changes of fatigability and muscle performance in severe obese patients after an integrated body mass reduction program. Diabetes Nutr Metab. 2003;16:88-93.
26. Krupp LB, LaRocca NG, Muir-Nash J, Steinberg AD. The fatigue severity scale. Application to patients with multiple sclerosis and systemic lupus erythematosus. Arch Neurol. 1989;46:1121-3.

27. Levinger I, Goodman C, Hare DL, et al. Psychological responses to acute resistance exercise in men and women who are obese. J Strength Cond Res. 2009;23:1548-52.

28. Paolillo FR, Milan JC, Bueno Pde G, et al. Effects of excess body mass on strength and fatigability of quadriceps in postmenopausal women. Menopause. 2012;19:556-61.

29. Capodaglio P, Castelnuovo G, Brunani A, et al. Functional limitations and occupational issues in obesity: a review. Int J Occup Saf Ergon. 2010;16:507-23.

30. Corbeil P, Simoneau M, Rancourt D, et al. Increased risk for falling associated with obesity: mathematical modeling of postural control. IEEE Trans Neural Syst Rehabil Eng. 2001;9:126-36.

31. Fjeldstad C, Fjeldstad AS, Acree LS, et al. The influence of obesity on falls and quality of life. Dyn Med. 2008;7:4.

32. de Rekeneire N, Visser M, Peila R, et al. Is a fall just a fall: correlates of falling in healthy older persons. The Health, Aging and Body Composition Study. J Am Geriatr Soc. 2003;51:841-6.

33. Messier SP. Diet and exercise for obese adults with knee osteoarthritis. Clin Geriatr Med. 2010;26:461-77.

34. Messier SP, Gutekunst DJ, Davis C, DeVita P. Weight loss reduces knee-joint loads in overweight and obese older adults with knee osteoarthritis. Arthritis Rheum. 2005;52:2026-32.

35. Runhaar J, Koes BW, Clockaerts S, Bierma-Zeinstra SM. A systematic review on changed biomechanics of lower extremities in obese individuals: a possible role in development of osteoarthritis. Obes Rev. 2011;12:1071-82.

36. Mazzuca SA, Brandt KD, Lane KA, Chakr R. Malalignment and subchondral bone turnover in contralateral knees of overweight/ obese women with unilateral osteoarthritis: implications for bilateral disease. Arthritis Care Res. 2011;63:1528-34.

37. Mork PJ, Holtermann A, Nilsen TI. Effect of body mass index and physical exercise on risk of knee and hip osteoarthritis: longitudinal data from the Norwegian HUNT Study. J Epidemiol Community Health. 2012;66:678-83.

38. Taylor ED, Theim KR, Mirch MC, et al. Orthopedic complications of overweight in children and adolescents. Pediatrics. 2006;117:2167-74.

39. Ekkekakis P, Lind E. Exercise does not feel the same when you are overweight: the impact of self-selected and imposed intensity on affect and exertion. Int J Obes (Lond). 2006;30:652-60. 